\title{
Hemoglobin-associated oxidative stress in the pericardial compartment of postoperative cardiac surgery patients
}

\author{
Philip A Kramer ${ }^{1,2,8}$, Balu K Chacko, ${ }^{1,2,8}$, Saranya Ravi ${ }^{1,2}$, Michelle S Johnson ${ }^{1,2}$, Tanecia Mitchell ${ }^{1,2}$, \\ Stephen Barnes ${ }^{2,3}$, Alireza Arabshahi ${ }^{3}$, Louis J Dell'Italia ${ }^{2,4,5}$, David J George ${ }^{6}$, Chad Steele ${ }^{7}$, James F George ${ }^{2,6}$, \\ Victor M Darley-Usmar ${ }^{1,2}$ and Spencer J Melby ${ }^{2,6}$
}

Atherosclerosis and valvular heart disease often require treatment with corrective surgery to prevent future myocardial infarction, ischemic heart disease, and heart failure. Mechanisms underlying the development of the associated complications of surgery are multifactorial and have been linked to inflammation and oxidative stress, classically as measured in the blood or plasma of patients. Postoperative pericardial fluid (PO-PCF) has not been investigated in depth with respect to the potential to induce oxidative stress. This is important because cardiac surgery disrupts the integrity of the pericardial membrane surrounding the heart and causes significant alterations in the composition of the pericardial fluid (PCF). This includes contamination with hemolyzed blood and high concentrations of oxidized hemoglobin, which suggests that cardiac surgery results in oxidative stress within the pericardial space. Accordingly, we tested the hypothesis that PO-PCF is highly pro-oxidant and that the potential interaction between inflammatory cell-derived hydrogen peroxide with hemoglobin is associated with oxidative stress. Blood and PCF were collected from 31 patients at the time of surgery and postoperatively from 4 to $48 \mathrm{~h}$ after coronary artery bypass grafting, valve replacement, or valve repair (mitral or aortic). PO-PCF contained high concentrations of neutrophils and monocytes, which are capable of generating elevated amounts of superoxide and hydrogen peroxide through the oxidative burst. In addition, PO-PCF primed naive neutrophils resulting in an enhanced oxidative burst upon stimulation. The PO-PCF also contained increased concentrations of cell-free oxidized hemoglobin that was associated with elevated levels of $F_{2 \alpha}$ isoprostanes and prostaglandins, consistent with both oxidative stress and activation of cyclooxygenase. Lastly, protein analysis of the PO-PCF revealed evidence of protein thiol oxidation and protein carbonylation. We conclude that PO-PCF is highly pro-oxidant and speculate that it may contribute to the risk of postoperative complications.

Laboratory Investigation (2015) 95, 132-141; doi:10.1038/labinvest.2014.144; published online 1 December 2014

In the United States there are $\sim 750000$ cardiac surgeries performed each year. ${ }^{1}$ Depending on the procedure, the incidence of postoperative complications such as atrial fibrillation is $15-50 \%$, which results in a prolonged hospital stay and an estimated $\$ 10000$ increase in hospital cost per occurrence. $^{2}$ Therapeutic strategies are only partially effective, and a lack of understanding of the complication has resulted in minimal improvement in treatment over the last several years. ${ }^{3,4}$ It has been documented that inflammatory markers are elevated in the serum of postoperative cardiac surgery patients and this correlates with increased occurrences of cardiac dysfunction and atrial fibrillation..$^{5-7}$ Some investigators have reported partial improvement in cardiac complications with systemic anti-inflammatory treatments. ${ }^{8}$ However, these studies have focused on serum concentrations of inflammatory molecules and have not evaluated the

\footnotetext{
'Department of Pathology, Mitochondrial Medicine Laboratory, University of Alabama at Birmingham, Birmingham, AL, USA; ${ }^{2}$ Department of Pathology, Center for Free Radical Biology, University of Alabama at Birmingham, Birmingham, AL, USA; ${ }^{3}$ Targeted Metabolomics and Proteomics Laboratory, Department of Pharmacology and Toxicology, University of Alabama at Birmingham, Birmingham, AL, USA; ${ }^{4}$ Division of Cardiovascular Sciences, Department of Medicine, Center for Heart Failure Research, University of Alabama at Birmingham, Birmingham, AL, USA; ${ }^{5}$ Department of Veterans Affairs Medical Center, Birmingham, AL, USA; ${ }^{6}$ Division of Cardiothoracic Surgery, Department of Surgery, University of Alabama at Birmingham, Birmingham, AL, USA and ${ }^{7}$ Division of Pulmonary, Allergy and Critical Care, Department of Medicine, University of Alabama at Birmingham, Birmingham, AL, USA

Correspondence: Dr SJ Melby, MD, Division of Cardiothoracic Surgery, Department of Surgery, University of Alabama at Birmingham, Birmingham, 35294 AL, USA. E-mail: Melbys@wudosis.wustl.edu

${ }^{8}$ These authors contributed equally to this work.

Received 17 July 2014; accepted 9 October 2014
} 
composition of the pericardial fluid, which may be more reflective of the local environment around the heart, especially for highly labile oxidation products and pro-inflammatory cytokines. Oxidative stress is known to exacerbate heart injury and can occur secondary to inflammation, surgical trauma, and cardiac ischemia., ${ }^{7,-11}$ Oxidative stress has been implicated in the development of atrial fibrillation immediately following surgery; however, the extent of its contribution and mechanisms responsible are yet to be elucidated. ${ }^{12-15}$

The pericardium is a specialized membrane surrounding the heart that performs specific physiological roles needed for cardiac function. ${ }^{16,17}$ In addition to acting as a lubricant, the pericardial fluid (PCF) contains several paracrine modulators, such as prostanoids, natriuretic peptides, and endothelins, which may regulate sympathetic tone, coronary vasomotor tone, heart rate, blood pressure, and complement responses. ${ }^{16,18}$ The pericardium is breached at the time of surgery, causing significant alterations in the PCF. In pericardial diseases, it has been shown that the composition and volume of postoperative PCF (PO-PCF) is altered and associated with cardiac dysfunction. ${ }^{19-23}$ Specifically, pericarditis, inflammation of the pericardium, has been shown to cause increased fluid volume and accumulation of inflammatory cells in the PCF and contributes to decreased cardiac function. These findings provide evidence that components of PCF can act as modulators of cardiomyocyte function despite the presence of the epicardium. ${ }^{24-27}$ In this study we tested the hypothesis that cardiac surgery results in the generation of highly pro-oxidant and pro-inflammatory molecules in the PCF and that these lead to oxidative damage in the pericardial environment.

\section{MATERIALS AND METHODS}

\section{Surgery, Blood Collection, and Cell Isolations}

All study protocols for the collection and handling of human samples were reviewed and approved by the Institutional Review Board, University of Alabama at Birmingham. Consent was obtained from adult ( $>19$ years old) patients undergoing cardiac surgery for ischemic heart disease or valvular heart disease: coronary artery bypass graft \pm aortic valve replacement/mitral valve repair/replacement, tricuspid valve repair, or replacement, or a valve procedure alone. Patients with ventricular assist devices, atrial fibrillation surgery, thoracic aorta surgery, non-cardiac surgery, and those with atrial fibrillation within 6 months were excluded from the study.

Blood samples (one to two tubes, $8.5 \mathrm{ml} /$ tube) were collected from 31 patients (22 male and 9 female patients, 62 ( \pm 26 ) years of age (Table 1 ) in vacutainers (BD Biosciences) containing $1.5 \mathrm{ml}$ ACD solution (trisodium citrate, $22.0 \mathrm{~g} / \mathrm{l}$; citric acid, $8.0 \mathrm{~g} / \mathrm{l}$; and dextrose $24.5 \mathrm{~g} / \mathrm{l}$ ) as anticoagulant and processed within $2 \mathrm{~h}$ of collection. Procedures were designed to prevent activation of the cells during isolation and were performed at room temperature unless otherwise specified. PO-PCF was obtained and assayed in parallel with peripheral blood drawn from the same patient at the time of the start of surgery, and $4,12,24$, and $48 \mathrm{~h}$ after the patient left the operating room. PO-PCF was allowed to accumulate no more than $1 \mathrm{~h}$ in the mediastinal drainage tube before collection. Cells were prepared from blood or PCF as described previously. ${ }^{28,29}$ Monocytes and neutrophils were pelleted, counted, and plated on an extracellular flux analysis plates for measurement of oxidative burst. Prepared cells were $\geq 95 \%$ viable as determined by Trypan Blue exclusion (data not shown).

\section{Measurement of Myoglobin, Troponin, and Creatine Kinase}

Cardiac injury markers were assessed by a Luminex beadbased multiplex assay using fluorescent bead technology (Milliplex kit, Millipore Corp.) in a 96-well format. Measurements were conducted in compliance with the manufacturer's instructions. Standard curves correlating the mean fluorescence intensity with concentration were generated for each analyte using the standards supplied by the manufacturer, and unknown values were calculated within the linear portion of the generated standard curves.

\section{Hemoglobin concentration}

The postoperative plasma and PCF were centrifuged at $1500 \mathrm{~g}$ for $10 \mathrm{~min}$ to remove cells and then at $5000 \mathrm{~g}$ for $10 \mathrm{~min}$ to

Table 1 Patient characteristics

\begin{tabular}{|c|c|c|c|c|c|c|}
\hline Age (years) & Weight (kg) & Height (cm) & BMI & Gender & Afib & Procedure \\
\hline \multirow[t]{3}{*}{$62.3( \pm 25.6)$} & $83.4( \pm 27.8)$ & $166.3( \pm 24.4)$ & $30.9( \pm 16.2)$ & M 71\% & $32 \%$ & CABG (pump) 19\% \\
\hline & & & & & & Valve $16 \%$ \\
\hline & & & & & & Valve + CABG 13\% \\
\hline
\end{tabular}

BMI, body mass index.

Table of the patient demographics involved in this study. The age, weight, height, BMl, gender, and presentation of atrial fibrillation after surgery were obtained from a total of 31 patients. Data presented as mean \pm s.d. 
remove cellular debris. Absorbance spectra from 500 to $700 \mathrm{~nm}$ was measured using a DU 800 spectrophotometer (BeckmanCoulter). Total hemoglobin was determined after subtracting the background absorbance at $700 \mathrm{~nm}$ from the isosbestic point for methemoglobin $(\mathrm{MetHb})$ and oxyhemoglobin $(\mathrm{OxyHb})$ at $523 \mathrm{~nm}$ with an extinction coefficient of 7.12 as reported by Snell and Marini. ${ }^{30}$ MetHb concentration was calculated using the extinction coefficient of $4.4 \mathrm{~mm}^{-1} \mathrm{~cm}^{-1}$ at $631 \mathrm{~nm} .{ }^{31}$ Assuming the two major species of hemoglobin, MetHb and $\mathrm{OxyHb}$, were present in the PCF, OxyHb was measured as the difference between $\mathrm{MetHb}$ and total hemoglobin.

\section{Flow Cytometry Analysis and Complete Blood Count in PCF}

PCF collected at the time of surgery and for the first $48 \mathrm{~h}$ after surgery was centrifuged and the buffy coat isolated as described above. Isolated leukocytes were separated by forward and side scatter and analyzed with flow cytometry using CD14-PE and CD16-APC to differentiate monocyte and neutrophil populations.

\section{Determination of Lipid Peroxidation Products with Mass Spectrometry}

Isoprostane and prostaglandin extractions of human serum and PCF were performed as described previously. ${ }^{32}$ Briefly, aliquots of patient PCF were stored frozen $\left(-80^{\circ} \mathrm{C}\right)$ and contained $1 \mathrm{~mm}$ BHT and $50 \mu \mathrm{M}$ EDTA and DTPA. Samples $(1 \mathrm{ml})$ were thawed and diluted to $3 \mathrm{ml}$ with $0.02 \mathrm{M}$ Bis-Tris, $\mathrm{pH} 6.0$ containing 8 -iso-PGF $2 \alpha-\mathrm{d} 4$ as an internal standard and $1 \mathrm{~mm}$ BHT and $50 \mu \mathrm{m}$ EDTA before being loaded on to prepared Strata X-AW $33 \mu$ polymeric weak anion exchange cartridges (Phenomenex, Torrance, CA, USA). Eluted samples were dried under a constant stream of nitrogen and reconstituted in methanol containing $1 \mathrm{~mm}$ BHT and $50 \mu \mathrm{M}$ EDTA. F2 $\alpha$-Isoprostanes and prostaglandins were measured by isotope dilution, reverse-phase liquid chromatographymultiple reaction ion-monitoring mass spectrometry as previously described. ${ }^{32}$

\section{Protein Thiol Oxidation}

To detect protein thiols, Bodipy-IAM (Bodipy-iodoacetamide; Invitrogen, Eugene, OR, USA), a fluorescently labeled alkylating agent capable of forming covalent adducts with cysteine thiol groups that are not involved in disulfide linkages was utilized. In brief, PCF samples from individual patients ( 0 and $4 \mathrm{~h}$ post surgery) were diluted 1:10 with de-ionized water before determining protein concentration using the Lowry DC protein assay (Bio-Rad Laboratories, Hercules, CA, USA). Subsequently, $5 \mu \mathrm{g}$ of PCF protein was treated with $500 \mu \mathrm{M}$ Bodipy-IAM for $30 \mathrm{~min}$ to assess protein thiol modifications. The reaction was stopped with $5 \times$ SDS-PAGE sample buffer $(1 \mathrm{M}$ Tris- $\mathrm{HCl}, \mathrm{pH} 6.8,10 \%$ SDS, 30\% glycerol, and $0.05 \%$ bromophenol blue) containing $5 \% \beta$-mercaptoethanol.

Samples were resolved using $12.5 \%$ SDS-PAGE gels and imaged in-gel using a Typhoon imager (GE Healthcare
Biosciences, Pittsburgh, PA, USA). Following imaging, gels were immediately stained with Coomassie Brilliant Blue G-250 (coomassie blue; Bio-Rad Laboratories) for $1 \mathrm{~h}$, destained overnight, and imaged using the AlphaView SA imager (Protein Simple, Santa Clara, CA, USA) to evaluate protein loading. The Bodipy-IAM fluorescent signal intensity for albumin was quantified using ImageQuantTL analysis software (GE Healthcare Biosciences) and the coomassie blue protein stain for albumin was quantified using the AlphaView SA software.

\section{Protein Carbonylation}

To determine the extent of protein carbonylation because of aldehyde/ketone moieties on proteins in PO-PCF $(0$ and $4 \mathrm{~h})$, samples were first depleted of albumin. PCF samples $(100 \mu \mathrm{l})$ were loaded on rehydrated SwellGel blue Resin (Thermo Scientific) and eluted as per the manufacturer's instructions. The protein content was measured in the flow-through material. Next, $100 \mu \mathrm{g}$ protein was incubated in the presence of $1 \mathrm{~mm}$ biotin hydrazide (Pierce) for $30 \mathrm{~min}$ at $37^{\circ} \mathrm{C}$. The reaction was stopped with the addition of $1 \mathrm{M}$ Tris- $\mathrm{HCl}, \mathrm{pH}$ 7.4, after which proteins were resolved by SDS-PAGE and transferred to nitrocellulose.

\section{Measurement of the Oxidative Burst}

Monocytes and neutrophils were isolated from peripheral blood and PCF at $4 \mathrm{~h}$ post surgery and compared with healthy donors. ${ }^{29}$ Where indicated, healthy donor neutrophils were pretreated for $1.5 \mathrm{~h}$ with the post centrifugation $(1500 \mathrm{~g})$ supernatant from the 4-h PO-PCF sample (0-10\% supernatant). After incubation, the oxidative burst was stimulated with phorbol 12-myristate 13-acetate (PMA, $100 \mathrm{ng} / \mathrm{ml}$ ), after the inhibition of mitochondrial respiration using antimycin A $(10 \mu \mathrm{M})$. The oxygen consumption rate was measured using an extracellular flux analyzer (Seahorse Bioscience) as previously described. ${ }^{29}$ The area under the curve (AUC) after PMA stimulation was subtracted from the rate of oxygen consumption after antimycin A treatment to give the total generation of reactive oxygen species (ROS) over a period of 24 or $48 \mathrm{~min}$. This oxygen consumption has previously been shown to be dependent on NADPH oxidase activity. ${ }^{29}$

\section{Statistics}

Each blood sample was analyzed with the number of replicates indicated and data are presented as mean \pm s.e.m. Statistical significance was determined using either Student's $t$-test or one-way ANOVA, as indicated, followed by Tukey's post hoc analysis, with $P \leq 0.05$ designated as statistically significant.

\section{RESULTS}

Cell-Free OxyHb and MetHb are Present in the PCF Surrounding the Heart after Cardiac Surgery and Followed by Increased Markers of Myocardial Damage

Following cardiac surgery, the pericardial membrane is left partially opened leading to the mixing of blood from lacerations, lipids from the myocardial, perivascular, and 
epicardial fat surrounding the heart and from the traumatized tissue and exuded fluids from the surgical wounds with the PCF in the pericardial space. Figure 1 illustrates a chest $\mathrm{CT}$ scan of a representative patient $12 \mathrm{~h}$ after surgery and depicts the location of the mediastinal drains and the effusion surrounding the pericardial space. These drains typically capture $100-300 \mathrm{ml}$ in the first few hours following surgery and then taper to about $<150 \mathrm{ml} / 24 \mathrm{~h}$ before the drains are removed. A major constituent of the postoperative PCF in the first few hours after surgery is blood from surgical trauma to the heart and vessels as well as non-cardiac sources. The proportion of blood gradually diminishes and is largely absent by $24 \mathrm{~h}$ post surgery in a normal postoperative course.

Continuous drainage of the PO-PCF is maintained up to $72 \mathrm{~h}$ post surgery. As shown in (Figure 1a), PCF accumulates around the heart even in the presence of mediastinal drainage tubes in place. Indicators of cardiac muscle injury following surgery, including myoglobin, troponin-I, and CK-MB
(Figure 1b), were present in the PO-PCF at significantly greater levels when compared with patient serum and increased in the PCF subsequent to elevated markers of oxidative stress. Following surgery, blood was present in the PCF and significant hemolysis was also detected. Figures $2 \mathrm{a}$ and $\mathrm{b}$ show the OxyHb and MetHb concentrations in PCF as determined by the visible absorbance spectra at $0-48 \mathrm{~h}$ post surgery, indicating significant hemolysis and oxidation of the cell-free hemoglobin. Shown in Figure $2 c$ is the quantification of the $(\mathrm{MetHb}) /(\mathrm{OxyHb})$ ratio in the intraoperative and postoperative samples. The ratio is significantly increased at $12 \mathrm{~h}$ and is consistent with a pro-oxidant environment.

\section{The Pro-Inflammatory Environment in the Postoperative PCF Promotes Monocyte and Neutrophil Recruitment} Flow cytometry analysis of the PCF and blood at $4-48 \mathrm{~h}$ showed two major populations of leukocytes. The predominant population, visible by $4 \mathrm{~h}$, was $\mathrm{CD} 14^{-} \mathrm{CD} 16^{+}$neutrophils, which

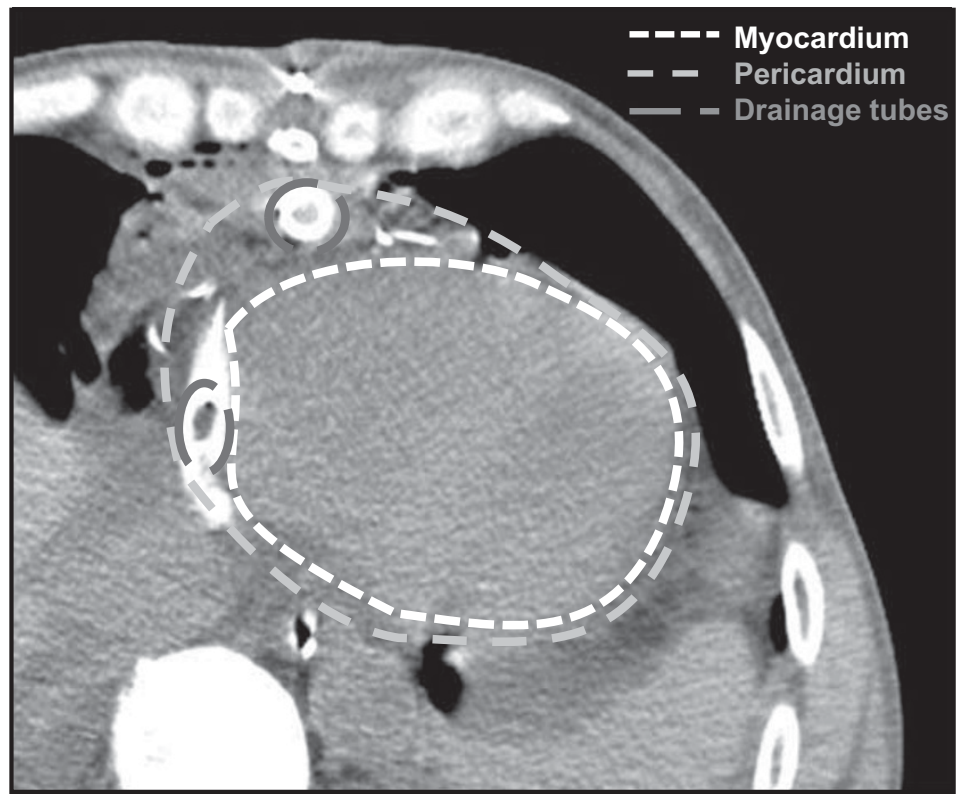

b
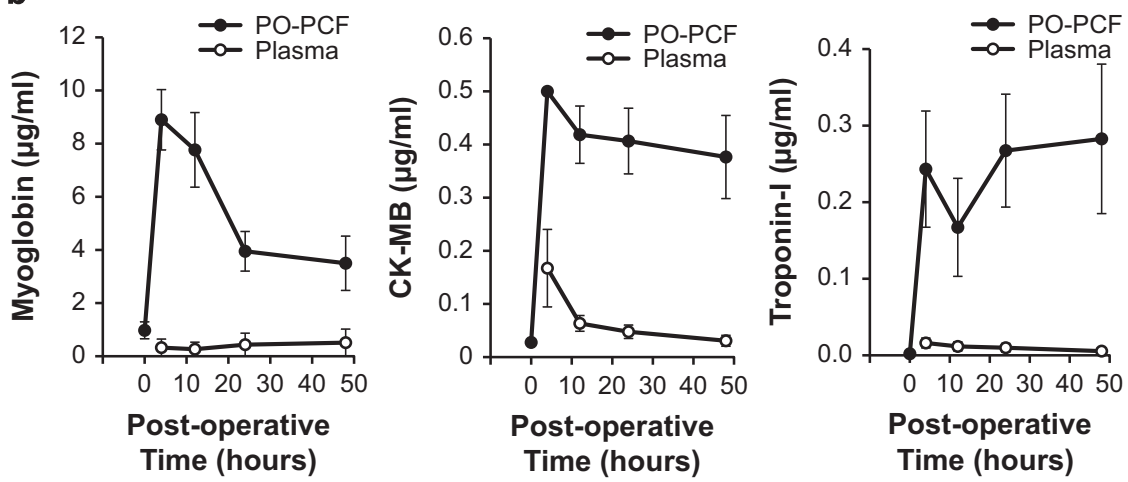

Figure 1 Postoperative pericardial fluid accumulates around the heart. (a) Postoperative axial CT scan image of a cardiac surgery patient demonstrates accumulation of pericardial fluid around the heart. (b) The cardiac injury markers, myoglobin, CK-MB, and troponin-I are significantly elevated in the postoperative pericardial fluid compared with their plasma controls $(P<0.05)$. 
a

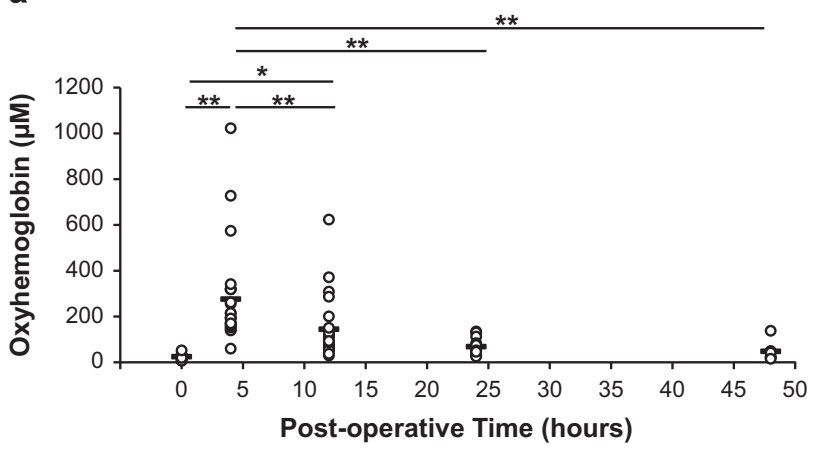

b

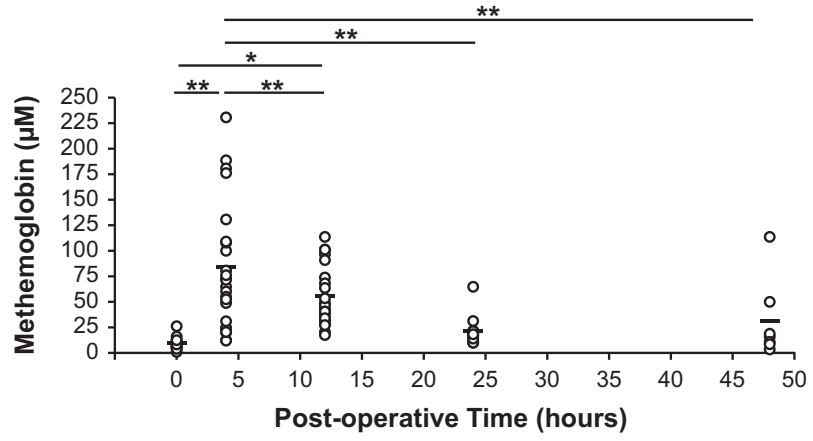

C

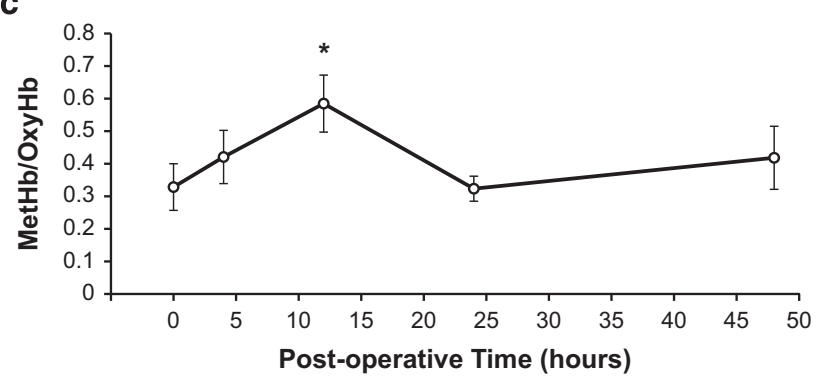

Figure 2 Hemoglobin and methemoglobin in postoperative PCF. Samples collected from the same patient during surgery $(0 \mathrm{~h})$ and post surgery $(4,12,24$, and $48 \mathrm{~h})$ were immediately centrifuged at $500 \mathrm{~g}$ for 15 min, supernatants collected, and centrifuged again to remove cells and large debris at $1500 \mathrm{~g}$ for $10 \mathrm{~min}$. (a) The oxyhemoglobin and (b) methemoglobin levels are visualized for each patient at the corresponding time after surgery as measured by spectrophotometry. (c) The methemoglobin/oxyhemoglobin ratio $(\mathrm{MetHb} / \mathrm{OxyHb})$ is shown to determine time points of relatively greater oxidative stress compared with the $0 \mathrm{~h}$ control. The mean values are represented by a horizontal bar. Data from 31 patients. ${ }^{*} P<0.05,{ }^{*} P<0.005$.

comprised $64.0 \pm 19.7 \%$ of cells found in the PCF by $12 \mathrm{~h}$ (Figure 3a). The next most abundant lineage was $\mathrm{CD} 14^{+}$ $\mathrm{CD} 6^{+}$monocytes that were $1.01 \pm 0.25 \%$ of cells at $12 \mathrm{~h}$ and increased to $6.02 \pm 2.53 \%$ of total leukocytes by $24 \mathrm{~h}$ (Figure 3a). The lineages of these cells were also confirmed by CD66b and MHCII expression (data not shown). Monocytes were detectable in the $0 \mathrm{~h}$ PCF as confirmed by a complete blood count performed on unprocessed PCF; however, counts did not significantly increase after the surgery (Figure $3 b$ ). Neutrophil counts were similar to monocytes at the com- a

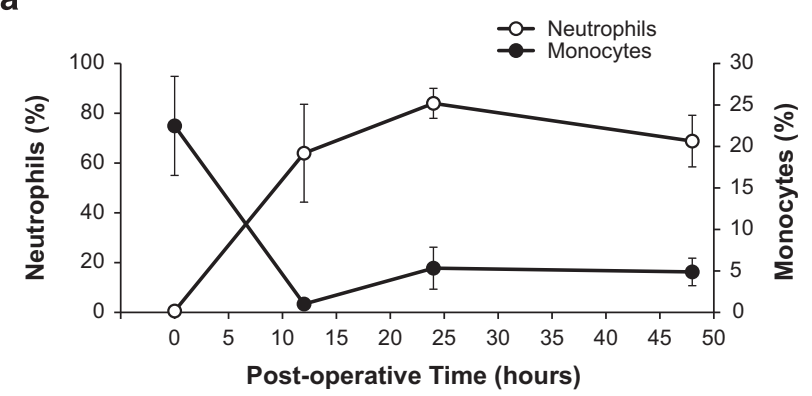

b
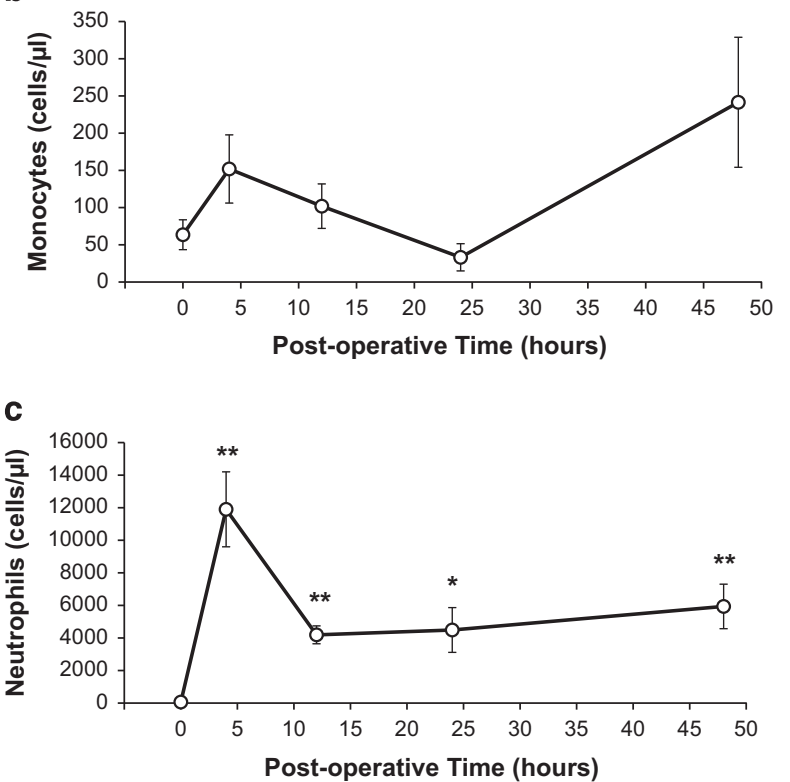

Figure 3 Flow cytometric analysis of the pericardial fluid (PCF). (a) The proportions of gated cells expressing CD14 and CD16 characteristic of neutrophils and monocytes, respectively, were enumerated at each time point. Note the differences in the $y$ axis scales for macrophages and neutrophils. (b) Absolute monocyte (c) and neutrophil counts were performed on unaltered PCF drawn during surgery $(0 \mathrm{~h})$ and post surgery $\left(4,12,24\right.$, and 48 h). Data reported as mean \pm s.e.m. ${ }^{*} P \leq 0.05,{ }^{* *} P<0.005$.

mencement of surgery but were significantly elevated after $4 \mathrm{~h}$ and for the duration of recovery (Figure 3c).

\section{The Postoperative Pericardial Environment Primes the Oxidative Burst in Monocytes and Neutrophils}

A major source of hydrogen peroxide production during inflammation is NADPH oxidase 2 (NOX2) in monocytes and neutrophils. Using the monocytes and neutrophils isolated from both PCF and blood, the potential maximal activity of the NADPH oxidase system was determined. We found that monocytes isolated from PCF exhibited an increased oxidative burst when stimulated by PMA $(100 \mathrm{ng} / \mathrm{ml})$ as compared with monocytes isolated from the peripheral blood of healthy age-matched controls (Figure 4a). No significant difference was seen between the oxidative burst of monocytes (Figures $4 \mathrm{a}$ and c, $P=0.15$ ) or neutrophils (Figures $4 \mathrm{~b}$ and $\mathrm{c}, P=0.33$ ) isolated from postoperative 
a

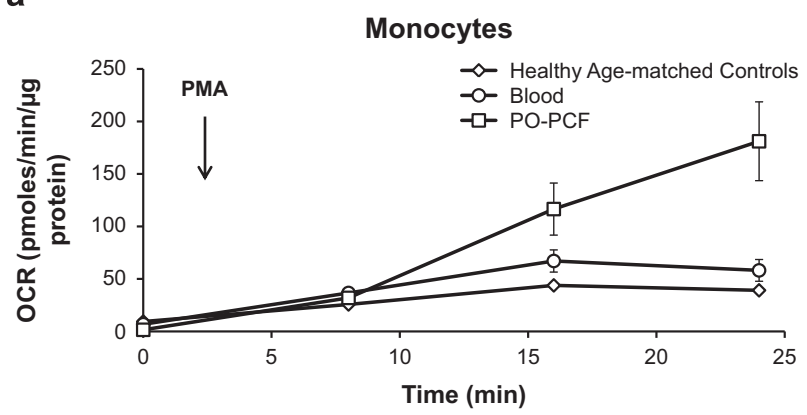

b

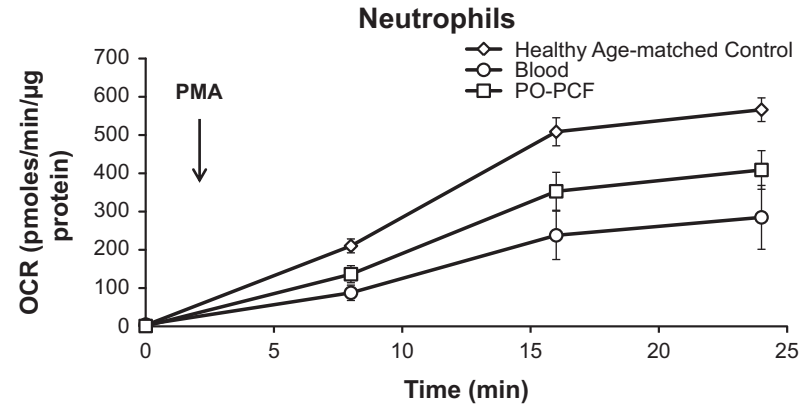

C

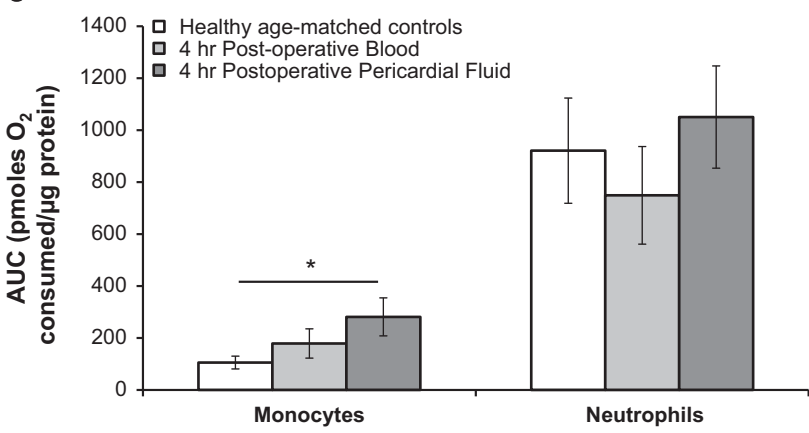

Figure 4 Priming of the oxidative burst of monocytes and neutrophils isolated from the blood and pericardial fluid of postoperative cardiac surgery patients. (a) Healthy age-matched control $(n=8)$ monocytes and (b) neutrophils isolated from freshly drawn blood were stimulated with phorbol 12-myristate 13-acetate (PMA; $100 \mathrm{ng} / \mathrm{ml}$ ). Oxygen consumption was monitored over time on a Seahorse Extracellular Flux analyzer and compared with cells collected from blood and pericardial fluid obtained at $4 \mathrm{~h}$ post surgery $(n=7)$. (c) The oxidative burst was measured as the area under the curve (AUC) of oxygen consumption over 24 min following PMA injection, and normalized to the oxygen consumption rate after antimycin A $(10 \mu \mathrm{m})$ injection to inhibit mitochondrial respiration. In all, $250 \mathrm{k}$ cells/well (monocytes) and $125 \mathrm{k}$ cells/well (neutrophils) were plated with three to five replicate wells per sample. Data reported as mean \pm s.e.m. ${ }^{*} P \leq 0.05$.

patient blood and PCF at $4 \mathrm{~h}$. However, to test the acute effect of PO-PCF on the priming of neutrophils to generate high levels of ROS, neutrophils from a healthy donor were incubated for $1.5 \mathrm{~h}$ with increasing concentrations of PO-PCF obtained from a patient at $4 \mathrm{~h}$ post surgery and then stimulated with PMA as described above. Quantitation of oxygen consumption demonstrated that PO-PCF exposure
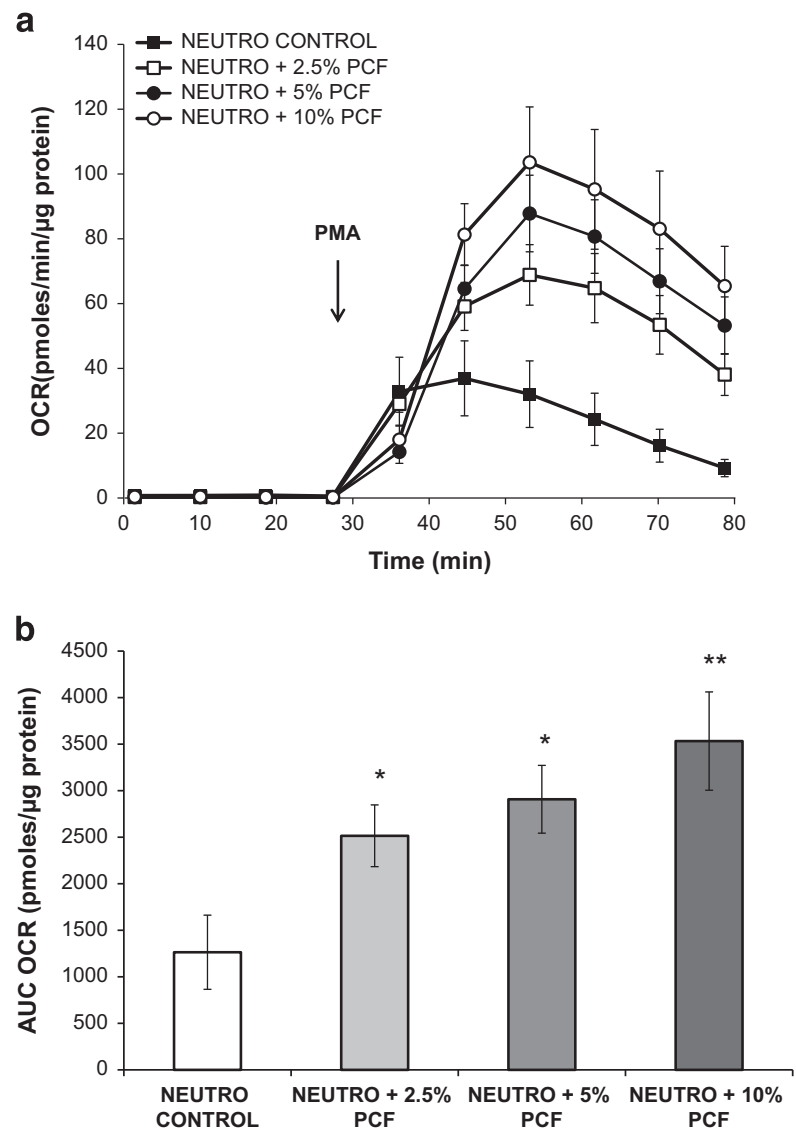

Figure 5 Postoperative pericardial fluid $(4 \mathrm{~h})$ primes neutrophils for increased oxidative burst. Pericardial fluid was centrifuged at $1500 \mathrm{~g}$ and then diluted to $2.5,5$, and $10 \%$ in XF DMEM. It was then added to freshly isolated and plated healthy donor neutrophils $1.5 \mathrm{~h}$ before the assay. (a) Baseline OCR was measured in neutrophils before inhibition of mitochondrial respiration with antimycin $\mathrm{A}(10 \mu \mathrm{m})$ and induction of the oxidative burst with phorbol 12-myristate 13-acetate (PMA, $100 \mathrm{ng} / \mathrm{ml}$ ). (b) The area under the curve (AUC) during the 48-min oxidative burst response was calculated and plotted as a function of PCF dose. ${ }^{*} P<0.05,{ }^{* *} P<0.005$. Three to five replicated wells per group.

primes the neutrophils to increase the production of superoxide and hydrogen peroxide compared with the untreated controls in a dose-dependent manner (Figures 5a and $\mathrm{b})$.

\section{Lipid Peroxidation Products Indicate a Pro-Oxidant Environment in the Postoperative PCF}

$\mathrm{MetHb}$ and myoglobin promote lipid peroxidation particularly in the presence of hydrogen peroxide. ${ }^{31,33}$ Mass spectrometry analysis revealed high and variable concentrations of the nonspecific lipid peroxidation products of arachidonic acid derived isoprostane 8 -iso- $\mathrm{PGF}_{2 \alpha}$ and its stereoisomer 8 -iso-15- PGF $_{2 \alpha}$ (F2 isoprostanes) in PCF at 4 and $12 \mathrm{~h}$ following surgery (Figure 6a). These concentrations returned to near basal levels by $24-48 \mathrm{~h}$. The prostaglandin $\mathrm{F}_{2 \alpha}$. (PGF2 $\alpha$ ) and its stereoisomer 15(R)-PGF $2 \alpha$ (Prostaglandins) showed a similar pattern, although levels were $\sim 10$-fold 
a

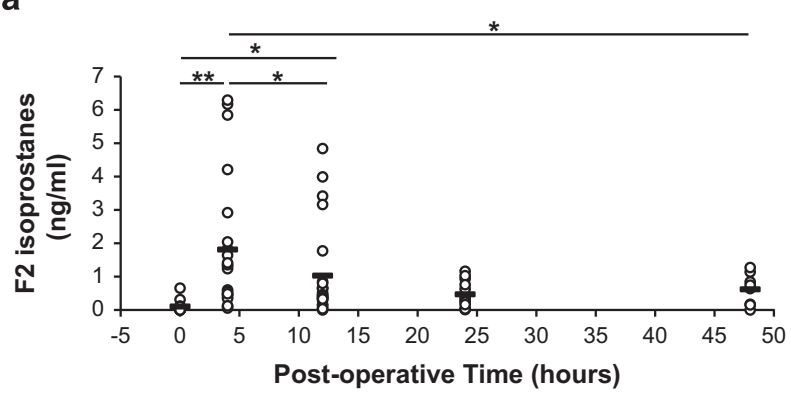

b

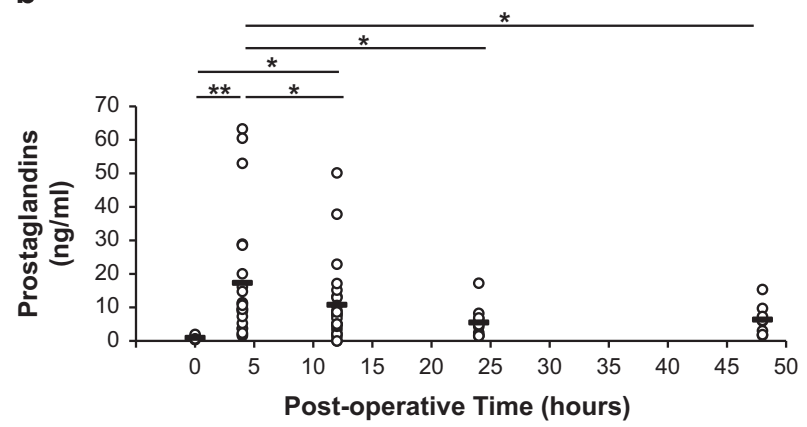

C

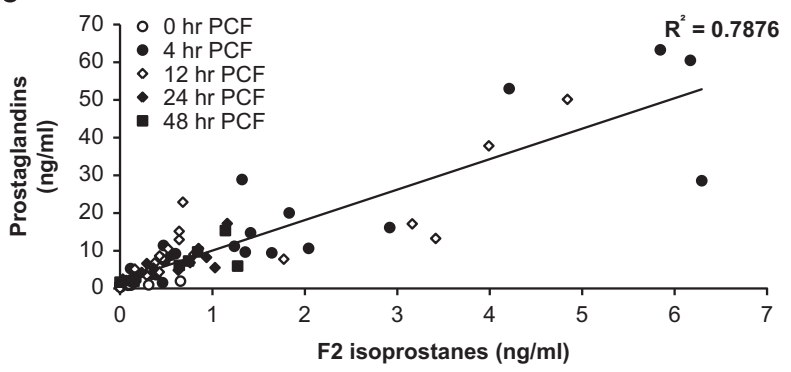

Figure 6 Quantitation of $\mathrm{F}_{2 \alpha}$ Isoprostanes and prostaglandins in postoperative PCF by LCMS/MS. Lipids were extracted from postoperative pericardial fluid using solid-phase extraction columns as described in the Materials and Methods and analyzed with mass spectrometry. (a) 8-isoprostaglandin $\mathrm{F} 2 \alpha$ concentrations and (b) prostaglandin $\mathrm{F} 2 \alpha$ concentrations were assessed in $0,4,12,24$, and 48 h postoperative PCF patient samples. (c) Regression analysis of $F_{2 \alpha}$ isoprostanes plotted against prostaglandins over all time points. Data represented as individual patient values plus with the mean represented by a horizontal bar at $0 \mathrm{~h}(n=13), 4 \mathrm{~h}(n=22), 12 \mathrm{~h}(n=23), 24 \mathrm{~h}(n=12)$, and $48 \mathrm{~h}$ $(n=8)$. Statistical significance was determined using one-way ANOVA followed by Tukey's post hoc analysis, with $P<0.05$ indicating statistical significance. ${ }^{*} P<0.05,{ }^{* *} P<0.005$.

higher than the isoprostanes consistent with the activation of cyclooxygenase (Figure 6b). Interestingly, the levels of isoprostanes were well correlated with prostaglandins (Figure 6c) suggesting that the available arachidonic acid, which is the substrate for both these lipid oxidation products, may also be increasing in PO-PCF.

\section{Protein Thiol Oxidation and Carbonyl Formation are Increased in Postoperative PCF}

Oxidants such as hydrogen peroxide and lipid peroxidation products are capable of modifying cysteine residues on pro- teins. To determine the extent of these reactions in the PCF, free thiol and carbonyl formation detection techniques were utilized. As shown in Figure 7a, Bodipy-IAM labeling of albumin, which has a single available thiol and is the predominant protein in both plasma and PO-PCF, was significantly decreased in the $4 \mathrm{~h}$ PO-PCF post-surgery samples compared with the $0 \mathrm{~h}$ PCF samples. Carbonylation of PCF proteins as determined by biotin hydrazide labeling of albumin detected significant carbonyl formation of proteins at $4 \mathrm{~h}$ post surgery compared with the intraoperative sample ( $0 \mathrm{~h}$ PO-PCF). These findings indicate that significant oxidative modification and adduct formation of proteins occurs following cardiac surgery (Figure 7b). Furthermore, these data are consistent with the presence of hemoglobin and isoprostanes observed at the same time points (Figures 2 and 6).

\section{DISCUSSION}

Alterations in the composition and volume of the PCF occur after cardiac surgery. These alterations include the accumulation of inflammatory cells and oxidative molecules, which can potentially lead to postoperative complications and alter cardiac function. As shown, these changes include elevated cardiac injury markers, increased levels of cell-free hemoglobin and methemoglobin, inflammatory cell infiltration, and the accumulation of lipid peroxidation products. Taken together, surgical trauma initiates red blood cell hemolysis, pro-inflammatory cell recruitment, and the active generation of pro-oxidative and pro-inflammatory molecules. It is likely that a major source of pro-oxidative molecules in the pericardial space post surgery is the neutrophil population that immigrates into the area in large numbers.

An established link between oxidant production and inflammation is the oxidative burst, a process by which inflammatory stimuli and antigens activate NADPH oxidase to produce superoxide and hydrogen peroxide as an innate host defense. Inflammatory cells are a source of superoxide and hydrogen peroxide as well as nitric oxide and hypochlorous acid. ${ }^{34-36}$ The oxidative burst is capable of generating these oxidative species in the micromolar range, which will oxidize MetHb to the ferryl form, an efficient initiator of lipid peroxidation. ${ }^{33,37-41}$ Inflammation has been found to be associated with atrial fibrillation, although most investigators have monitored the systemic inflammatory markers rather than the cardiac compartment in which inflammation directly occurs. ${ }^{42,43}$ Recently, Pinho-Gomes et al. ${ }^{44}$ demonstrated an association between ROS in atrial tissue and the onset of postoperative atrial fibrillation after cardiac surgery providing evidence that ongoing myocardial injury from oxidative stress may contribute to postoperative complications such as atrial fibrillation.

The postoperative pericardial environment is lipid-rich, given the proximity of epicardial and pericardial fat. ${ }^{45,46}$ The enzymatic (cyclooxygenase-mediated) and non-enzymatic (heme-protein-mediated) oxidation of polyunsaturated fatty acids generates biologically significant oxidation products that 
a

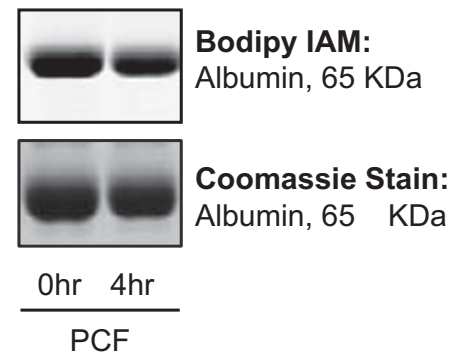

b

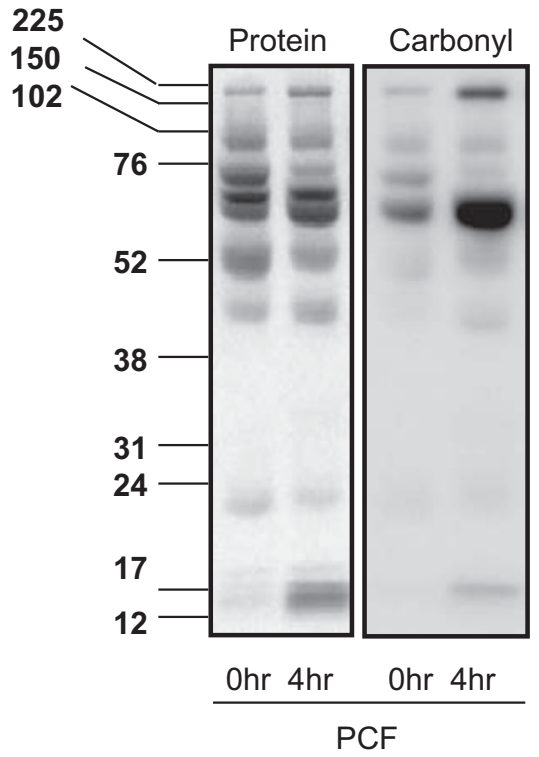

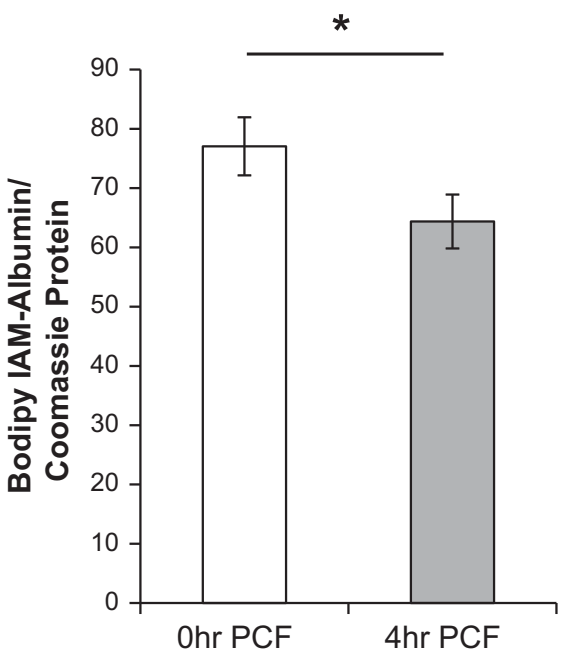

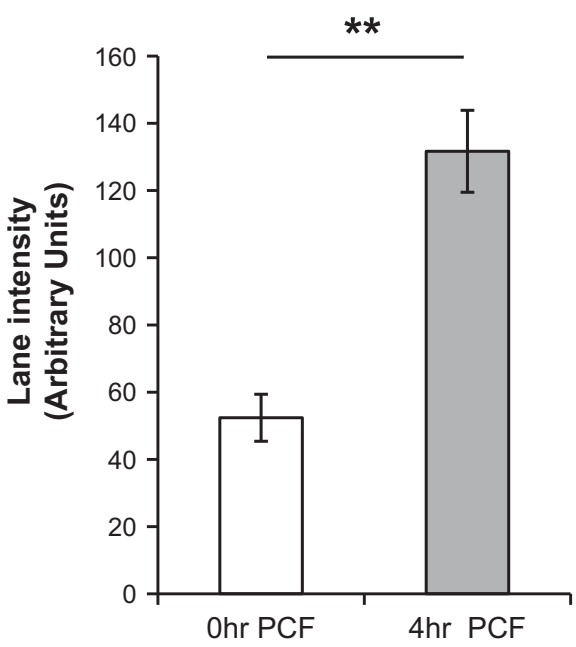

Figure 7 Oxidative modifications to proteins in postoperative PCF. (a) Representative in-gel fluorescence image of individual patient PCF (0 and $4 \mathrm{~h}$ ) treated with $500 \mu \mathrm{m}$ Bodipy-iodoacetamide (Bodipy-IAM) for $30 \mathrm{~min}$ to assess protein thiol modifications, where lower fluorescence intensity represents increased oxidized protein thiols. Following alkylation, $5 \mu \mathrm{g}$ of protein was resolved by $12.5 \%$ SDS-PAGE and imaged in-gel using a Typhoon imager. The bottom panel shows coomassie blue stain to confirm equal protein loading. Quantitation of the Bodipy-IAM fluorescence signal for albumin normalized to coomassie blue protein staining; $n=7$ patients. Data expressed as mean \pm s.e.m. ${ }^{*} P<0.05$ compared with 0 h PCF. (b) Carbonylated proteins $(100 \mu \mathrm{g})$ from 0 - and 4-h albumin-depleted PCF were derivatized with biotin hydrazide $(1 \mathrm{~mm})$ for 30 min at $37^{\circ} \mathrm{C}$. Reactions were stopped with $1 \mathrm{M}$ Tris- $\mathrm{HCl}, \mathrm{pH} 7.4$ and proteins $(10 \mu \mathrm{g})$ separated using SDS-PAGE. The extent of carbonylation was determined with western blot analysis using Streptavidin. The quantification of lane intensities is shown to the right.; $n=4$ patient matched sets. Statistical significance was measured using Student's $t$-Test. Data expressed as mean \pm s.e.m. ${ }^{*} P<0.05,{ }^{*} P<0.005$.

have a role in inflammation, signaling, and cell damage. Lipid peroxidation products have also been linked to the oxidative stress observed in ischemic heart disease ${ }^{47-51}$ and might also contribute to postoperative cardiac dysfunction.

In this study, the PO-PCF showed high concentrations of oxidized hemoglobin and evidence of lipid peroxidation, which has been shown to be a quantitative marker of oxidant stress and has previously been associated with increased incidence of heart failure. ${ }^{52}$ The formation of lipid radicals ultimately results in bioactive and/or electrophilic lipids capable of modifying nucleophilic protein residues such as cysteine, tyrosine, arginine, and histidine. Consistent with oxidative protein modification, the available thiol pool on albumin was significantly decreased at $4 \mathrm{~h}$ post surgery in PCF. Isoprostanes, a common product of non-enzymatic arachidonic acid peroxidation, are commonly used markers of oxidative stress in vivo and were present at elevated levels in the PCF at postoperative time points 4 and $12 \mathrm{~h}$ (Figure 6). Although the pericardial drains serve the purpose of limiting postoperative tamponade, the cardiac tissues are still exposed 
to an effusion containing high levels of oxidative molecules that can contribute to postoperative cardiac dysfunction, possibly including electromechanical disturbances, which are characteristic of atrial fibrillation. This concept is further supported by data from animal models demonstrating that oxidant production in the heart can lead to disruption of gap junctions and arrhythmic events, the blockade of ROS resulted in dramatic decreases in arrhythmias. ${ }^{53}$

Oxidative stress has been shown to contribute to worse patient outcomes following cardiac surgery. ${ }^{54,55}$ Traditionally, these markers were measured by the presence of reactive lipids such as malondialdehyde and HNE as well as the presence of antioxidant enzymes such as catalase. ${ }^{56}$ Using the thiol alkylating reagent iodoacetamide linked to the fluorophore Bodipy, we showed that albumin in the PO-PCF contained significantly fewer available thiols when compared with pre-operative PCF. In addition, we examined protein carbonylation, a marker of oxidative protein modification in intraoperative and postoperative PCF samples. Consistent with other indices of oxidative stress, the amount of protein carbonylation in postoperative PCF was significantly increased compared with intraoperative PCF (Figure 7). These data support increased production of reactive lipids and increased oxidant production in the PCF following surgery.

Superoxide production has an important role in ROSmediated inflammatory signaling. ${ }^{57-61}$ Under basal conditions, NADPH oxidase activity remains low; however, under stimuli such as increased pro-inflammatory cytokines, enzymatic activity can be increased by a process known as priming. ${ }^{62-66}$ Under chronic levels of low stimuli, phagocytic cells such as neutrophils can be primed, resulting in a heightened response to exogenous stimuli such as PMA. We determined whether monocytes and neutrophils isolated from the postoperative pericardial space or treated with PCF were primed for oxidant production by stimulation with PMA. When stimulated, monocytes from the pericardial space showed a much higher capacity for oxidative burst when compared with blood monocytes isolated from the same patient and age-matched controls. Using neutrophils isolated from healthy donors, we showed a dose-dependent oxidative burst capacity in response to priming with increasing amounts of $4 \mathrm{~h} \mathrm{PO-PCF} \mathrm{(Figure} \mathrm{5).}$ These data clearly demonstrate that monocytes and neutrophils, in response to the postoperative PCF milieu, are primed for oxidant production, supporting the concept that oxidative stress in the pericardium following surgery is in part due to the infiltrating inflammatory cells.

In summary, these data show that postoperative PCF contains high levels of cell-free hemoglobin, isoprostanes, and prostaglandins, which were not found in concurrent sampling of blood serum from the same patients or in the intraoperative PCF. We also demonstrate that a highly prooxidant environment is generated in the postoperative PCF of cardiac surgery patients. Evidence supports that this prooxidant milieu surrounding the heart may trigger postoperative atrial fibrillation. The incomplete drainage of this effusion after surgery may not be sufficient to prevent oxidative injury to myocytes. Additional studies are required to investigate whether therapies to inhibit oxidative stress in the pericardium may improve outcomes by preventing the onset or severity of atrial fibrillation. These data suggest an interesting relationship between oxidant production by infiltrating leukocytes, MetHb, and lipid peroxidation products and encourage larger-scale trials to determine the nature and mechanism of this relationship and its potential role in the development of postoperative complications.

\section{ACKNOWLEDGMENTS}

We thank Gloria A Benavides for her valuable technical assistance. This work was supported by T32HL07918 (PAK), P30DK056336 (BC), DK079337 (VDU,SB), HL103859 (SWB), T32HL007457 (TM), UAB CCTS pilot grant award (SJM), Birmingham Veterans Administration Merit Review grants (LJD), and the UAB-UCSD O'Brien Core Center for Acute Kidney Injury Research (DK079337). The mass spectrometer used in these studies was purchased with an award (SB) from the UAB Health Services Foundation General Endowment Fund.

\section{DISCLOSURE/CONFLICT OF INTEREST}

The authors declare no conflict of interest.

1. Popovic JR. 1999 National Hospital Discharge Survey: annual summary with detailed diagnosis and procedure data. Vital Health Stat 2001; (151), i-v: 1-206.

2. Aranki SF, Shaw DP, Adams DH, et al. Predictors of atrial fibrillation after coronary artery surgery. Current trends and impact on hospital resources. Circulation 1996;94:390-397.

3. Parkash R, Verma A, Tang AS. Persistent atrial fibrillation: current approach and controversies. Curr Opin Cardiol 2010;25:1-7.

4. Mathew ST, Patel J, Joseph S. Atrial fibrillation: mechanistic insights and treatment options. Eur J Internal Med 2009;20:672-681.

5. Gedikli O, Dogan A, Altuntas I, et al. Inflammatory markers according to types of atrial fibrillation. Int J Cardiol 2007;120:193-197.

6. Chelazzi C, Villa G, De Gaudio AR. Postoperative atrial fibrillation. ISRN Cardiol 2011;2011:203179.

7. Aldhoon B, Melenovsky V, Peichl P, et al. New insights into mechanisms of atrial fibrillation. Physiol Res 2010;59:1-12.

8. Imazio M, Brucato A, Ferrazzi P, et al. Colchicine reduces postoperative atrial fibrillation: results of the colchicine for the prevention of the postpericardiotomy syndrome (COPPS) atrial fibrillation substudy. Circulation 2011;124:2290-2295.

9. Elahi MM, Flatman S, Matata BM. Tracing the origins of postoperative atrial fibrillation: the concept of oxidative stress-mediated myocardial injury phenomenon. Eur J Cardiovasc Prev Rehabil 2008;15:735-741.

10. Rodrigo R, Castillo R, Cereceda M, et al. Non-hypoxic preconditioning of myocardium against postoperative atrial fibrillation: mechanism based on enhancement of the antioxidant defense system. Med Hypotheses 2007;69:1242-1248.

11. Aronson D, Boulos M, Suleiman A, et al. Relation of C-reactive protein and new-onset atrial fibrillation in patients with acute myocardial infarction. AM J Cardiol 2007;100:753-757.

12. Lappegard KT, Hovland A, Pop GA, et al. Atrial fibrillation: inflammation in disguise? Scand J Immunol 2013;78:112-119.

13. Fontes ML, Mathew JP, Rinder HM, et al. Atrial fibrillation after cardiac surgery/cardiopulmonary bypass is associated with monocyte activation. Anesth Analg 2005;101:17-23.

14. Chen MC, Chang JP, Liu WH, et al. Increased inflammatory cell infiltration in the atrial myocardium of patients with atrial fibrillation. Am J Cardiol 2008;102:861-865.

15. Ishii $\mathrm{Y}, \mathrm{Sch}$ essler RB, Gaynor SL, et al. Inflammation of atrium after cardiac surgery is associated with inhomogeneity of atrial conduction and atrial fibrillation. Circulation 2005;111:2881-2888.

16. Dudzinski DM, Mak GS, Hung JW. Pericardial diseases. Curr Prob Cardiol, 37:75-118. 
17. Zipes DP. Atrial fibrillation: a review of pathophysiology. Semin Interv Cardiol 1997;2:203-213.

18. Bodson L, Bouferrache K, Vieillard-Baron A. Cardiac tamponade. Curr Opin Crit Care 2011;17:416-424.

19. Karatolios K, Pankuweit S, Maisch B. Diagnostic value of biochemical biomarkers in malignant and non-malignant pericardial effusion. Heart Failure Rev. 2013;18:337-344.

20. Ben-Horin S, Shinfeld A, Kachel E, et al. The composition of normal pericardial fluid and its implications for diagnosing pericardial effusions. Am J Med 2005;118:636-640.

21. Selmeci L, Antal M, Horkay F, et al. Enhanced accumulation of pericardial fluid ferritin in patients with coronary artery disease. Coron Artery Dis 2000;11:53-56

22. Gibson AT, Segal MB. A study of the composition of pericardial fluid with special reference to the probable mechanism of fluid formation. $J$ Physiool 1978;277:367-377.

23. Eisalo A, Konttinen A. Composition of pericardial fluid in cholesterol pericarditis. Acta Med Scand 1972;191:125-128.

24. Megged O, Argaman Z, Kleid D. Purulent pericarditis in children: is pericardiotomy needed? Pediatr Emerg Care 2011;27:1185-1187.

25. Sparano DM, Ward RP. Pericarditis and pericardial effusion: management update. Curr Treat Options Cardiovasc Med 2011;13:543-555.

26. Katz LH, Pitlik S, Porat E, et al. Pericarditis as a presenting sign of infective endocarditis: two case reports and review of the literature. Scand J Infect Dis 2008:40:785-791.

27. Troughton RW, Asher CR, Klein AL. Pericarditis. Lancet 2004;363:717-727.

28. Kramer PA, Chacko BK, Ravi $\mathrm{S}$, et al. Bioenergetics and the oxidative burst: protocols for the isolation and evaluation of human leukocytes and platelets. J Vis Exp 2014:85:e51301.

29. Chacko BK, Kramer PA, Ravi S, et al. Methods for defining distinct bioenergetic profiles in platelets, lymphocytes, monocytes, and neutrophils, and the oxidative burst from human blood. Lab Invest 2013;93:690-700.

30. Snell SM, Marini MA. A convenient spectroscopic method for the estimation of hemoglobin concentrations in cell-free solutions. J Biochem Biophys Methods 1988;17:25-33.

31. Antonini E, Brunori M. Hemoglobin and Myoglobin in their Reactions with Ligands, Amsterdam, 1971, p 436.

32. Prasain JK, Arabshahi A, Taub PR, et al. Simultaneous quantification of F2-isoprostanes and prostaglandins in human urine by liquid chromatography tandem-mass spectrometry. J Chromatogr B Analyt Technol Biomed Life Sci 2013:913-914:161-168.

33. Moore KP, Holt SG, Patel RP, et al. A causative role for redox cycling of myoglobin and its inhibition by alkalinization in the pathogenesis and treatment of rhabdomyolysis-induced renal failure. J Biol Chem 1998:273:31731-31737.

34. Chedid P, Hurtado-Nedelec M, Marion-Gaber B, et al. Adiponectin and its globular fragment differentially modulate the oxidative burst of primary human phagocytes. Am J Pathol 2011;180:682-692.

35. Santos SS, Brunialti MK, Rigato O, et al. Generation of nitric oxide and reactive oxygen species by neutrophils and monocytes from septic patients and association with outcomes. Shock 2012;38:18-23.

36. Jukic $T$, Ihan A, Jukic D. Tetrahydrophthalazine derivative 'sodium nucleinate' exert its anti-inflammatory effects through inhibition of oxidative burst in human monocytes. Coll Antropol 2012;36:409-412.

37. Heyn J, Beiras-Fernandez A, Luchting B, et al. Inflammatory reactions and hydrocortisone in the setting of cardiac surgery: an overview. Cardiovasc Hematol Agents Med Chem 2011;9:56-61.

38. Fink R, Al-Obaidi M, Grewal $\mathrm{S}$, et al. Monocyte activation markers during cardiopulmonary bypass. Perfusion 2003;18:83-86.

39. Franke $\mathrm{A}$, Lante $\mathrm{W}$, Fackeldey $\mathrm{V}$, et al. Proinflammatory and antiinflammatory cytokines after cardiac operation: different cellular sources at different times. Ann Thorac Surg 2002;74:363-370, discussion 370-361.

40. Edmunds Jr. LH. Inflammatory response to cardiopulmonary bypass. Ann Thorac Surg 1998;66(5 Suppl):S12-S16.

41. Khare S, Bhutani LK, Rao DN. Modulation of peripheral blood derived monocytes/macrophages from leprosy patients using 'tuftsin' for production of reactive oxygen intermediates. Lepr Rev 1993;64:208-218.

42. Abdelhadi $\mathrm{RH}$, Gurm HS, Van Wagoner DR, et al. Relation of an exaggerated rise in white blood cells after coronary bypass or cardiac valve surgery to development of atrial fibrillation postoperatively. Am J Cardiol 2004;93:1176-1178.

43. Lo B, Fijnheer R, Nierich AP, et al. C-reactive protein is a risk indicator for atrial fibrillation after myocardial revascularization. Ann Thorac Surg 2005;79:1530-1535.

44. Pinho-Gomes AC, Reilly S, Brandes RP, et al. Targeting inflammation and oxidative stress in atrial fibrillation: role of 3-hydroxy-3methylglutaryl-coenzyme a reductase inhibition with statins. Antioxid Redox Signal 2014;20:1268-1285.

45. Guzzardi MA, lozzo P. Fatty heart, cardiac damage, and inflammation. Rev Diabet Stud 2011;8:403-417.

46. Sacks HS, Fain JN, Cheema $P$, et al. Depot-specific overexpression of proinflammatory, redox, endothelial cell, and angiogenic genes in epicardial fat adjacent to severe stable coronary atherosclerosis. Metab Syndr Relat Disord 2011;9:433-439.

47. Dincic $D$, Jovic $P$, Obradovic $S$, et al. Lipid peroxidation intensity and lipid status parameters in the estimation of the severity of ischemic heart disease. Vojnosanitetski Pregled 1998;55:359-367.

48. Chandra $M$, Chandra N, Agrawal $R$, et al. The free radical system in ischemic heart disease. Int J Cardiol 1994;43:121-125.

49. Higdon A, Diers AR, Oh JY, et al. Cell signalling by reactive lipid species: new concepts and molecular mechanisms. Biochem J 2012;442:453-464.

50. Higdon AN, Landar A, Barnes $\mathrm{S}$, et al. The electrophile responsive proteome: integrating proteomics and lipidomics with cellular function. Antioxid Redox Signal 2012;17:1580-1589.

51. Eaton P, Hearse DJ, Shattock MJ. Lipid hydroperoxide modification of proteins during myocardial ischaemia. Cardiovasc Res 2001:51:294-303.

52. Mallat Z, Philip I, Lebret $M$, et al. Elevated levels of 8-iso-prostaglandin F2alpha in pericardial fluid of patients with heart failure: a potential role for in vivo oxidant stress in ventricular dilatation and progression to heart failure. Circulation 1998:97:1536-1539.

53. Sovari AA, Rutledge CA, Jeong EM, et al. Mitochondria oxidative stress, connexin43 remodeling, and sudden arrhythmic death. Circ Arrhythm Electrophysiol 2013;6:623-631.

54. Karu I, Taal G, Zilmer K, et al. Inflammatory/oxidative stress during the first week after different types of cardiac surgery. Scand Cardiovasc J 2010;44:119-124.

55. McDonald Cl, Fraser JF, Coombes JS, et al. Oxidative stress during extracorporeal circulation. Eur J Cardiothorac Surg 2014:46:937-943.

56. Vukasovic JL, Moraga F, Diaz-Araya G, et al. Oxidative stress in pericardial fluid and plasma and its association with ventricular function. Int J Cardiol 2005:101:197-201.

57. Maulik SK, Kumar S. Oxidative stress and cardiac hypertrophy: a review. Toxicol Mech Methods 2012;22:359-366.

58. Nguyen Dinh Cat A, Montezano AC, Burger D, et al. Angiotensin II, $\mathrm{NADPH}$ oxidase, and redox signaling in the vasculature. Antioxid Redox Signal 2013;19:1110-1120.

59. Zhang M, Perino A, Ghigo A, et al. NADPH oxidases in heart failure: poachers or gamekeepers? Antioxid Redox Signal 2013;18:1024-1041.

60. Shen GX. Mitochondrial dysfunction, oxidative stress and diabetic cardiovascular disorders. Cardiovasc Hematol Disord Drug Targets 2012;12:106-112.

61. Hafstad AD, Nabeebaccus AA, Shah AM. Novel aspects of ROS signalling in heart failure. Basic Res. Cardiol. 2013;108:359.

62. Koval'chuk LV, Klebanov Gl, Ribarov SR, et al. Priming of phagocytes by cytokines and water-soluble products of lipid peroxidation. Biomed Sci 1991;2:221-231.

63. Kharazmi A, Nielsen $\mathrm{H}$, Rechnitzer $\mathrm{C}$, et al. Interleukin 6 primes human neutrophil and monocyte oxidative burst response. Immunol Lett 1989;21:177-184.

64. Hurtado-Nedelec M, Makni-Maalej K, Gougerot-Pocidalo MA, et al. Assessment of priming of the human neutrophil respiratory burst. Methods Mol Biol 2014;1124:405-412.

65. Finkel TH, Pabst MJ, Suzuki $H$, et al. Priming of neutrophils and macrophages for enhanced release of superoxide anion by the calcium ionophore ionomycin. Implications for regulation of the respiratory burst. J Biol Chem 1987;262:12589-12596.

66. El-Benna J, Dang PM, Gougerot-Pocidalo MA. Priming of the neutrophil NADPH oxidase activation: role of p47phox phosphorylation and NOX2 mobilization to the plasma membrane. Semin Immunopathol 2008;30:279-289. 